Article

\title{
Vortex Laser Based on a Plasmonic Ring Cavity
}

\author{
Xingyuan Wang ${ }^{1, *}$, Xiaoyong $\mathrm{Hu}^{2, *}$ and Tianrui Zhai ${ }^{3, *}$ (D) \\ 1 College of Mathematics and Physics, Beijing University of Chemical Technology, Beijing 100029, China \\ 2 State Key Laboratory for Mesoscopic Physics, Department of Physics, Collaborative Innovation Center of \\ Quantum Matter, Peking University, Beijing 100871, China \\ 3 School of Physics and Optoelectronics, Faculty of Science, Beijing University of Technology, \\ Beijing 100124, China \\ * Correspondence: wang_xingyuan@mail.buct.edu.cn (X.W.); xiaoyonghu@pku.edu.cn (X.H.); \\ trzhai@bjut.edu.cn (T.Z.)
}

check for

updates

Citation: Wang, X.; Hu, X.; Zhai, T Vortex Laser Based on a Plasmonic Ring Cavity. Crystals 2021, 11, 901. https://doi.org/10.3390/ cryst11080901

Academic Editor: Anna

Paola Caricato

Received: 19 June 2021

Accepted: 29 July 2021

Published: 31 July 2021

Publisher's Note: MDPI stays neutral with regard to jurisdictional claims in published maps and institutional affiliations.

Copyright: (c) 2021 by the authors. Licensee MDPI, Basel, Switzerland. This article is an open access article distributed under the terms and conditions of the Creative Commons Attribution (CC BY) license (https:// creativecommons.org/licenses/by/ $4.0 /)$.

\begin{abstract}
The orbital angular momentum (OAM) of the structure light is viewed as a candidate for enhancing the capacity of information processing. Microring has advantages in realizing the compact lasers required for on-chip applications. However, as the clockwise and counterclockwise whispering gallery modes (WGM) appear simultaneously, the emitted light from the normal microring does not possess net OAM. Here, we propose an OAM laser based on the standing-wave WGMs containing clockwise and counterclockwise WGM components. Due to the inhomogeneous intensity distribution of the standing-wave WGM, the single-mode lasing for the OAM light can be realized. Besides, the OAM of the emitted light can be designed on demand. The principle and properties of the proposed laser are demonstrated by numerical simulations. This work paves the way for exploring a single-mode OAM laser based on the plasmonic standing-wave WGMs at the microscale, which can be served as a basic building block for on-chip optical devices.
\end{abstract}

Keywords: micro-nano photonics devices; optical microcavity; micro-nano vortex laser; orbital angular momentum; plasmonic devices

\section{Introduction}

After the knowledge about the linear momentum and spin angular momentum of light, a breakthrough is the recognition of the orbital angular momentum (OAM) of light [1]. The OAM is a completely new degree of freedom. In theory, the OAM light provides infinite orthogonal modes. As a result, the OAM states can greatly expand the information capacity, which has directed attention to applications for optical communication [2] and quantum information [3]. Since the first demonstration of a laser in 1960 [4], various lasers have been investigated, such as the plasmonic nanolaser [5], the random laser [6-8], the photonic crystal nanocavity laser [9], and the exciton-polariton laser [10], and so forth. Advances in nano-technology make it possible to manipulate the light on a chip [11-15]. Because the generation of micro-scale OAM light is of great significance to on-chip applications, it has received extensive attention.

Optical vortex beams with helical phase front carry OAM. In order to obtain OAM light, one can modulate the wave front of a coherent light. The wave-front modulations are mostly based on changing the optical path difference, employing a geometry phase, or controlling diffraction on demand with suitable optical elements, such as spiral phase plates, lens, Pancharatnam-Berry phase elements, and holograph [16-23] and so forth. In addition, the direct generation of OAM light within the laser cavity has been developed, to the great attention of researchers [24-34]. Microring has advantages in realizing compact lasers. However, the conventional microring cavity supports the standing-wave WGM with the same weight of clockwise (CW) and counterclockwise (CCW) components, resulting in a net zero OAM of the emitted light. In order to obtain emitted light with OAM, much attention is paid to breaking the rotation symmetry between the two counter-propagating 
WGMs in the ring cavity. For instance, the OAM laser can be made based on the travelingwave WGM at the non-Hermitian exceptional point of a ring cavity [34]. Despite these impressive advances, designing controllable single-mode OAM lasers at the microscale are still challenging.

Here, we propose a vector OAM laser based on the plasmonic standing-wave WGMs. In this framework, the OAM light is constructed by modulating the scattered waves from the standing-wave WGM in the plasmonic ring cavity, and the desired single-mode lasing can be achieved.

\section{Principle and Results}

Figure 1a shows the schematic of an OAM laser. The ring cavity is a coaxial cylindrical structure with a silver-InGaAsP-silver geometry. The bottom of the cavity is encapsulated by silver, so only the upper face of the ring is reserved as the port of the OAM-light emission. This microcavity supports plasmonic mode, for which the electric field component with polarization along the radial direction in the cavity plane ( $x-y$ plane) is dominant. We construct the OAM light based on the standing-wave WGMs. For the standing wave, the oscillations between two adjacent nodes are in phase, while the oscillations on the opposite sides of a node are in anti-phase, that is, there is a $\pi$ phase jump at each node. However, the OAM light beam possesses helical phase fronts. In order to obtain OAM light, we introduce tiny effective-refractive-index perturbations at discrete positions along the azimuthal direction of the InGaAsP ring (the effective-refractive-index perturbations are in the region with $r_{\text {inner }} \leq r \leq r_{\text {outer }}$ and $0 \leq z \leq h_{\text {InGaAsP, }}$ where $r_{\text {inner }}, r_{\text {outer }}$ and $h_{\text {InGaAsP }}$ are the inner radius, outer radius, and height of the InGaAsP ring, respectively). The tiny effective-refractive-index perturbations will couple the standing-wave WGM to the OAM mode by extracting the phase of the standing wave and shifting it to the phase of the OAM mode. In detail, we introduce $2 m$ effective-refractive-index perturbations at discrete parts in the ring. Each individual section of the effective-refractive-index perturbations has an angular width $\Delta \theta=\pi / 2 m$ along the azimuthal direction of the ring. The azimuthal coordinates at the centrals of these $2 m$ sections are $\theta_{1}(n)=\pi n / m(n=1,2,3, \ldots, 2 m)$, respectively. For convenience, we define these $2 m$ sections of the ring as region 1 , and define other parts of the ring as region 2 (region 2 of the ring also consists of $2 m$ sections, where each individual section has an angular width $\Delta \theta$, and the azimuthal coordinates at the centrals of these sections are $\theta_{2}(n)=\pi n / m+\Delta \theta(n=1,2,3, \ldots, 2 m)$, respectively). In this framework, the standing-wave WGM with azimuthal mode number $l=m$ can be coupled to the desired OAM light. The effective refractive indexes in the ring $\left(r_{\text {inner }} \leq r \leq\right.$ $\left.r_{\text {outer, }} 0 \leq z \leq h_{\text {InGaAsP }}\right)$ along the azimuthal direction $(\theta)$ are

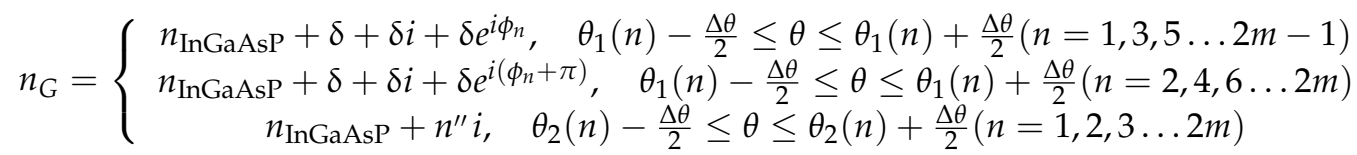

where the tiny perturbations $\delta e^{i \phi_{n}}$ and $\delta e^{i\left(\phi_{n}+\pi\right)}$ will couple the standing-wave WGMs with $l=m$ into the desired OAM light. Because the oscillations of the standing-wave WGMs jump a $\pi$ phase at each amplitude node, a $\pi$ is needed in the term $\delta e^{i\left(\phi_{n}+\pi\right)}$. $\phi_{n}=q n \pi / m(n=1,2,3, \ldots, 2 m)$. The OAM carried by the emitted light is related to $q(q=0, \pm 1, \pm 2, \ldots)$. The $\phi_{n}$ and $\phi_{n}+\pi$ can be controlled by tuning the real and imaginary part of the perturbation terms. Researchers have explored some methods to modulate the effective dielectric constant or effective refractive index [34,35]. The loss $n^{\prime \prime} i$ can be used to suppress the lasing of the competing modes. It is worth noting that the $\delta+\delta i$ is not required. However, in order to facilitate the practical effective refractive index modulations, $\delta+\delta i$ is used in our theoretical model. In this case, $\delta+\delta i+\delta e^{i \phi_{n}}$ and $\delta+\delta i+\delta e^{i\left(\phi_{n}+\pi\right)}(\delta>0)$ can be achieved without resorting to gain and negative real parts. In this paper, we adopt the following definition: the material corresponds to loss material when the imaginary part of the refractive index of the material is positive. 


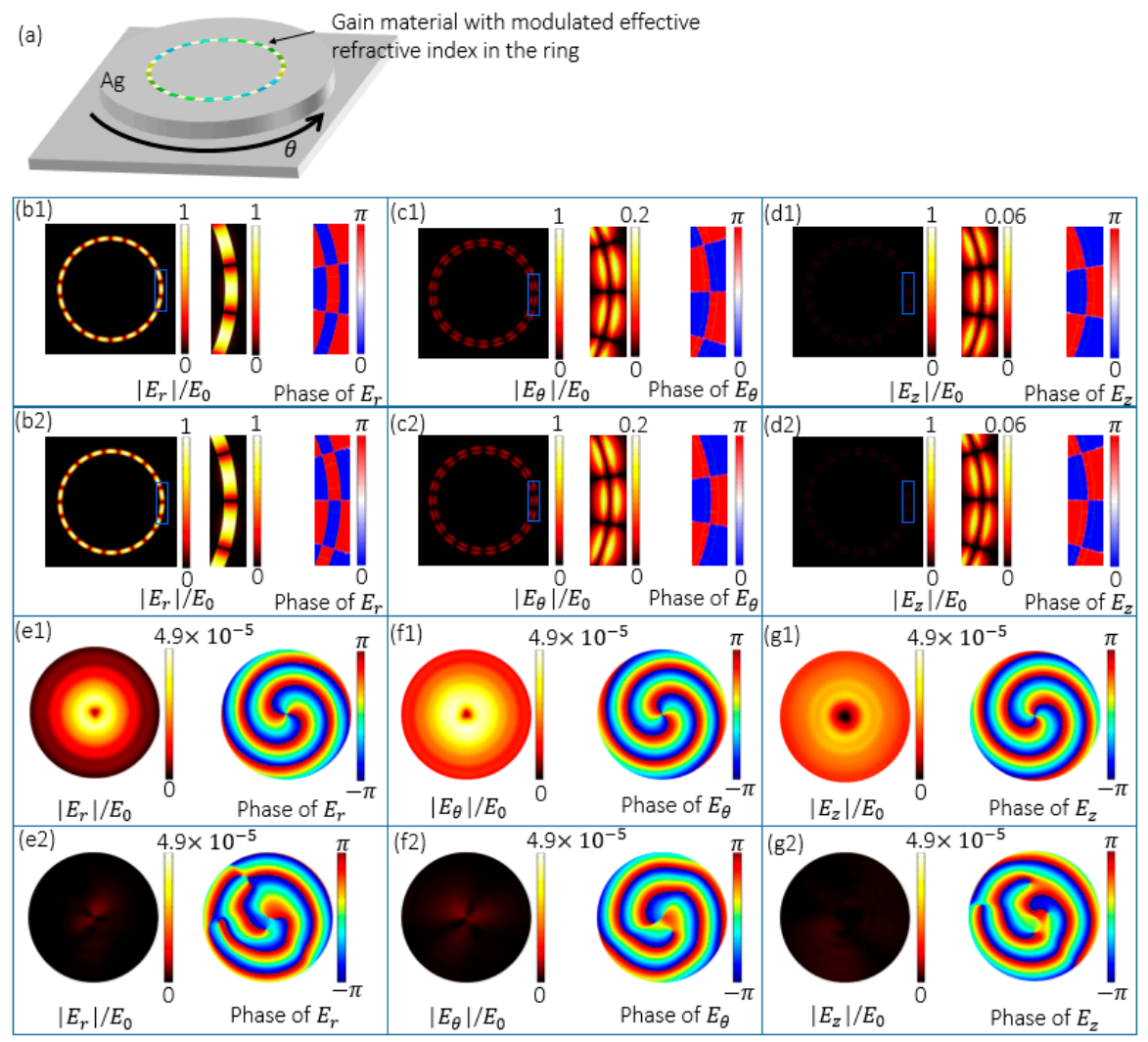

Figure 1. The cavity mode and the emitted mode of the OAM laser for $l=11$. (a) Schematic of an OAM laser. The $z=0$ is at the bottom of the InGaAsP ring, and the $(x=0, y=0)$ is at the center of the ring. (b1)-(g1), mode 1 inside the cavity [(b1)-(d1)] and the corresponding emission mode [(e1)-(g1)]. (b2)-(g2). Mode 2 inside the cavity [(b2)-(d2)] and the corresponding emission mode $[(\mathbf{e} 2)-(\mathbf{g} 2)]$. (b1),(b2), the intensity distributions $\left|E_{r}\right| / E_{0}$ of the radial component of the electric field inside the cavity (the $\left|E_{r}\right| / E_{0}$ and the phase of $E_{r}$ in the area marked by rectangular box is also presented in the enlarged figures). (c1),(c2), the intensity distributions $\left|E_{\theta}\right| / E_{0}$ of the azimuthal component of the electric field inside the cavity (the $\left|E_{\theta}\right| / E_{0}$ and the phase of $E_{\theta}$ in the area marked by rectangular box is also presented in the enlarged figures). (d1),(d2), the intensity distributions $\left|E_{z}\right| / E_{0}$ of the $z$-component of the electric field inside the cavity (the $\left|E_{z}\right| / E_{0}$ and the phase of $E_{z}$ in the area marked by rectangular box is also presented in the enlarged figures). (b1)-(d1), (b2)-(d2) are the mode properties at $z=h_{\text {InGaAsP }} / 2$. (e1)-(g1), (e2)-(g2) are in the cross section (the radius of the area is $5000 \mathrm{~nm}$ ) of the emission modes at $2 \mu \mathrm{m}$ above the upper surface of the cavity. $E_{0}$ is the maximum of the $\left|E_{r}\right|$ in the (b1). The green lines in the enlarged figures are the boundaries of different sections. The $q=2$ is used. The eigenfrequencies of the two modes are $1.9999 \times 10^{14}+i 6.25 \times 10^{11} \mathrm{~Hz}$ and $2.0031 \times 10^{14}+i 9.45 \times 10^{11} \mathrm{~Hz}$, respectively. These results are calculated by the Comsol Multiphysics.

Now, we carry out 3D full wave simulations (via the commercial software COMSOL Multiphysics) to demonstrate the OAM laser. We take a standing-wave WGM with $l=11$ in the microcavity as an example. The effective-refractive-index perturbations in region 1 consist of $2 m$ discrete sections with an angular width $\Delta \theta=\pi / 2 m$, where $m=11$. The azimuthal coordinate at the center of each section of region 1 is $\theta_{1}(n)=n \pi / 11$, where $n=1,2,3, \ldots, 22$. Then, the effective refractive indexes in the ring are

$$
n_{G}=\left\{\begin{array}{c}
n_{\text {InGaAsP }}+0.006+0.006 i+0.006 e^{i \phi_{n}}, \quad \frac{n \pi}{11}-\frac{\Delta \theta}{2} \leq \theta \leq \frac{n \pi}{11}+\frac{\Delta \theta}{2}(n=1,3,5 \ldots 21) \\
n_{\text {InGaAsP }}+0.006+0.006 i+0.006 e^{i\left(\phi_{n}+\pi\right)}, \quad \frac{n \pi}{11}-\frac{\Delta \theta}{2} \leq \theta \leq \frac{n \pi}{11}+\frac{\Delta \theta}{2}(n=2,4,6 \ldots 22) \\
n_{\text {InGaAsP }}+n^{\prime \prime} i, \quad \frac{n \pi}{11}+\frac{\Delta \theta}{2} \leq \theta \leq \frac{n \pi}{11}+\frac{3 \Delta \theta}{2}(n=1,2,3 \ldots 22)
\end{array}\right.
$$

where $\phi_{n}=q n \pi / 11, n_{\text {InGaAsP }}=3.34$, and $n^{\prime \prime}=0.012$. In the simulations, we consider the condition that the silver is at a low temperature, and use the refractive index 
$n_{\mathrm{Ag}}=0.0014+10.9741 i$ at $4.5 \mathrm{~K}$ [5], which has low metal loss. In this paper, we adopt the following definition: the mode corresponds to loss mode when the imaginary part of the eigenfrequency of the mode is positive. The inner radius, width and height of the InGaAsP ring are $620 \mathrm{~nm}, 50 \mathrm{~nm}$ and $200 \mathrm{~nm}$, respectively.

Figure 1 presents the details of the standing waves in the cavity and the emitted modes for $l=11$. Here, the laser cavity with $q=2$ is taken as an example, and the z-direction mode number and radial mode number of the cavity mode are fixed. The $\left|E_{r}\right|,\left|E_{z}\right|$, and $\left|E_{\theta}\right|$ show obvious nodes along the azimuthal direction of the ring [Figure 1(b1)-(d1),(b2)-(d2)], which is the typical character of the standing wave modes. Besides, the standing-wave nature of the cavity modes is further confirmed by the phases of $E_{r}, E_{z}$, and $E_{\theta}$ of the cavity modes (the last figures of the Figure 1(b1)-(d1),(b2)-(d2)). Because the cavity mode is coupled into the emitted OAM light by the tiny perturbations in region 1, mode 1 and mode 2 with different distributions in the cavity exhibit distinct emission characteristics. For mode 1, the azimuthal antinodes of $E_{r}$ (the dominant component of the electric field) match the sections of region 1 (Figure 1(b1),(d1)). Besides, the phase of the $E_{r}$ of mode 1 in each individual section of region 1 is the same, and the $E_{r}$ of mode 1 has a phase difference $\pi$ between adjacent sections of region 1 (Figure 1(b1)). Thus, mode 1 is coupled to the desired OAM mode as shown by Figure 1(e1)-(g1), in which the $E_{r}, E_{z}$ and $E_{\theta}$ all show phase characteristics of the OAM light. For mode 2, only the azimuthal antinodes of $E_{\theta}$ match the sections of region 1 (Figure 1(c2)), while the azimuthal antinodes of $E_{r}$ and $E_{z}$ match the sections of region 2 [Figure 1(b2),(d2)]. Besides, the $E_{r}, E_{\theta}$, and $E_{z}$ of mode 2 in each individual section of region 1 all simultaneously contain mode oscillations with opposite phases. Thus, the emission from mode 2 to the OAM mode is suppressed as shown in Figure 1(e2)-(g2) (the intensity of mode 1 and mode 2 in the cavity is nearly the same. However, compared with the bright color of the emitted light from mode 1 (Figure 1(e1)-(g1)), the emitted light from mode 2 (Figure 1(e2)-(g2)) shows a nearly dark color, indicating the suppression of the emitted light from mode 2.) This property will help suppress unwanted components in the emitted light.

Figure 2 shows that the OAM of the emitted light can be tuned by tuning the tiny modulation of the effective refractive index (tuning $q$ in the $\phi_{n}$ ). The emission modes for $q=1,2$, and 3 are presented in the first, second and third row of Figure 2, respectively. It can be found that the phase repeats one, two, and three times from 0 to $2 \pi$ upon one full circle around the center of the emitted light beam for $q=1,2$, and 3, respectively, which means the OAM can be designed on demand.

Now, we show that this OAM laser can naturally ensure the single-mode operation of desired mode. Here, we make a comparison between the modes with the same field distribution in the vertical cross section of the bend waveguide of the microring but with different field distributions in the azimuthal direction. For the standing-wave WGM, one mode (mode 1 ) of the pair of modes with $l=m$ is mainly confined in region 1 , and the other mode (mode 2 ) is mainly confined in region 2 . In addition, the cavity modes with $l \neq m$ all have nearly the same distribution weight in two regions. Thus, the desired mode 1 experiences the lower loss from region 2 than other modes, that is, enhancing the loss in region 2 can relatively suppress the lasing of other competing modes. In order to demonstrate this principle, we conduct the 3D full-wave simulations. We take the laser cavity with $q=2$ and $m=11$ as an example. Figure 3a shows the ratios of the volume integral of $|E|^{2}$ confined in region 1 to the volume integral of $|E|^{2}$ confined in region 2. Obviously, the electric fields of the desired mode 1 are mainly confined in region $1\left(\Gamma_{1} / \Gamma_{2} \sim 4.2\right)$. In contrast, the electric fields of mode 2 are mainly confined in region $2\left(\Gamma_{1} / \Gamma_{2} \sim 1 / 4.2\right)$. For other WGMs (for instance the mode pair with $l=10$ and mode pair with $l=12$.), electric fields are nearly equally distributed in region 1 and region 2 $\left(\Gamma_{1} / \Gamma_{2} \sim 1\right)$. Thus, as the loss in region 2 increases, the quality factor of mode 1 (red line in Figure $3 b$ ) decreases slower than other modes (blue line and black lines in Figure 3b). As a result, the single-mode lasing of the desired mode will be benefited when the perturbation 
loss of region 2 is relatively large. It is worth noting that the OAM natures of the emission mode will not change as the loss in region 2 increases as shown in Figure 3c.

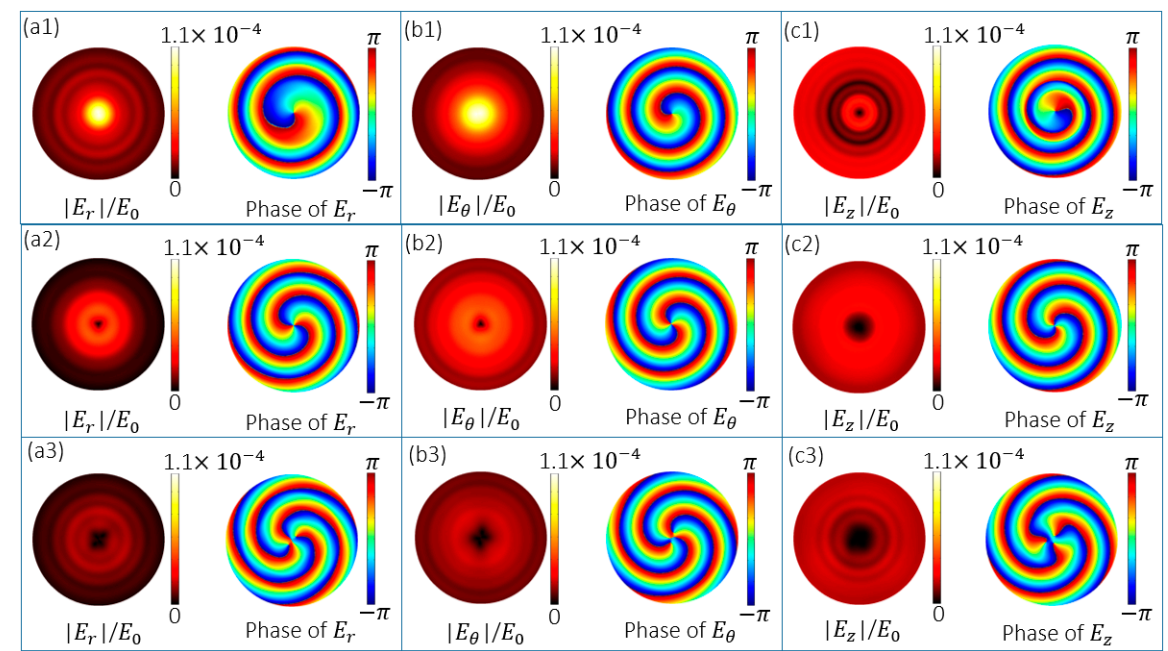

Figure 2. Tuning the OAM of the emitted light. The first row [(a1)-(c1)], second row [(a2)-(c2)], and third row $[(\mathbf{a} 3)-(\mathbf{c} 3)]$ of the figure present the simulation results of the modes for $q=1,2$ and 3 , respectively. These results are in the cross section (the radius of the area is $5000 \mathrm{~nm}$ ) of the emission modes at $2 \mu \mathrm{m}$ above the upper surface of the cavity. It is worth noting that the corresponding modes inside the cavity (mode 1) have nearly the same field intensity.

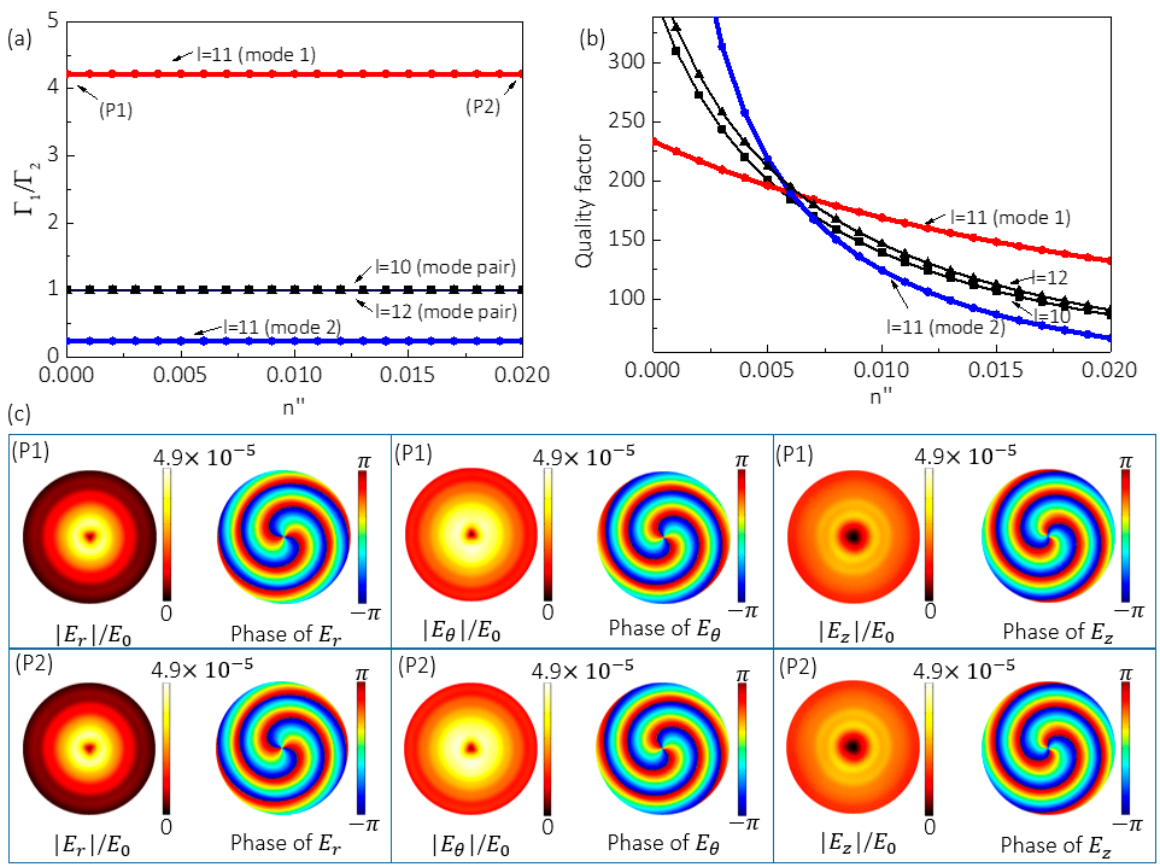

Figure 3. Suppressing the lasing of the competing modes. (a) The $n^{\prime \prime}$ dependence of the ratios of the volume integral of $|E|^{2}$ confined in the region 1 to the volume integral of $|E|^{2}$ confined in region 2, which is calculated as $\Gamma_{1} / \Gamma_{2}=\iiint_{\text {Region } 1}|E|^{2} d v / \iiint_{\text {Region } 2}|E|^{2} d v$. Here, $n^{\prime \prime}$ is the modulation in Equation (2). (b) The quality factors of the modes as a function of the $n^{\prime \prime}$. (c) The intensity $\left(\left|E_{r}\right| / E_{0}\right.$, $\left|E_{\theta}\right| / E_{0}$, and $\left|E_{z}\right| / E_{0}$ ) and phase distributions of the emission mode at P1 and P2 of the (a). The (c) are in the cross section (the radius of the area is $5000 \mathrm{~nm}$ ) of the emission mode at $2 \mu \mathrm{m}$ above the upper surface of the cavity. In this example, $q=2$ and $m=11$. The red lines correspond to the desired mode 1 with $l=11$, the blue lines correspond to mode 2 with $l=11$, the black lines correspond to the modes with $l=10$, and 12 . 
Finally, we investigate the switch of the OAM of the emitted light. In order to control the OAM of the emitted light, we modulate the effective refractive index of region 1 to couple one standing-wave WGM (mode 1) to an OAM mode, and modulate the effective refractive index of region 2 to couple the other standing-wave WGM (mode 2) to the other OAM mode. Then, the losses of region 1 and region 2 are used to select the lasing mode between mode 1 and mode 2 . Here, the effective refractive indexes in the ring are

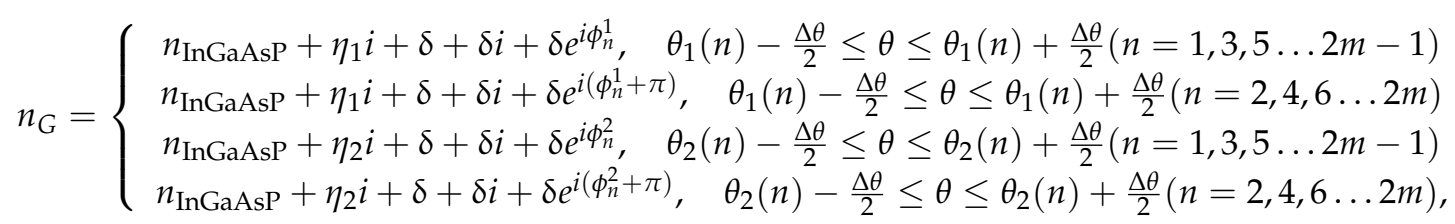

where $\Delta \theta=\pi / 2 m, \theta_{1}(n)=\pi n / m(n=1,2,3, \ldots, 2 m), \theta_{2}(n)=\pi n / m+\Delta \theta(n=1,2,3, \ldots, 2 m)$, $\phi_{n}^{1}=q_{1} n \pi / m(n=1,2,3, \ldots, 2 m), \phi_{n}^{2}=q_{2} n \pi / m(n=1,2,3, \ldots, 2 m)$. The electric field $|E|$ of mode 1 and mode 2 are mainly localized in region 1 and region 2, respectively. Thus, the lasing of mode 1 or mode 2 can be selectively suppressed by tuning the additional material loss $\left(\eta_{1} i\right)$ in region 1 and the additional material loss $\left(\eta_{2} i\right)$ in region 2, which enables the switch of the emission mode between two different OAM modes. Now, we present the results of the 3D full-wave simulation. In our example, we used the parameters $m=11, \delta=0.006, q_{1}=2, q_{2}=3$. We assume that the parameters $\left(\eta_{1}, \eta_{2}\right)$ are tuned from $\left(\eta_{1}=0, \eta_{2}=0.006\right)$ to $\left(\eta_{1}=0.006, \eta_{2}=0\right)$. The gain of the InGaAsP can be achieved when being pumped. The homogeneous pumping gain of the InGaAsP ring is mimicked by adding an additional background imaginary part $\Delta i(\Delta<0)$ to the effective refractive index of the InGaAsP ring. It is worth noting that we do not consider the effect of the specific gain spectrum of the gain material for the sake of simplicity. Figure 4a shows that the loss of mode 1 is compensated for before the loss of mode 2 with the increase of the homogeneous pumping gain of the InGaAsP for $\left(\eta_{1}=0, \eta_{2}=0.006\right)$. Thus, mode 1 will be the lasing mode. In this case, the phases of $E_{r}, E_{z}$ and $E_{\theta}$ of the emitted lasing beam all repeat two times from 0 to $2 \pi$ upon one full circle around the center of the emitted light beam (Figure $4 \mathrm{c})$. After tuning the system parameters $\left(\eta_{1}, \eta_{2}\right)$ from $\left(\eta_{1}=0, \eta_{2}=0.006\right)$ to $\left(\eta_{1}=0.006, \eta_{2}=0\right)$, the loss of mode 2 is compensated before the loss of mode 1 with the increase of the homogeneous pumping gain of the InGaAsP as shown by Figure $4 \mathrm{~b}$. Thus, the lasing mode switches from mode 1 to mode 2 . In this case, the emitted lasing beam is switched to the other OAM mode as shown in Figure 4d, in which the phases of the $E_{r}, E_{z}$ and $E_{\theta}$ all repeat three times from 0 to $2 \pi$ upon one full circle around the center of the emitted light. 

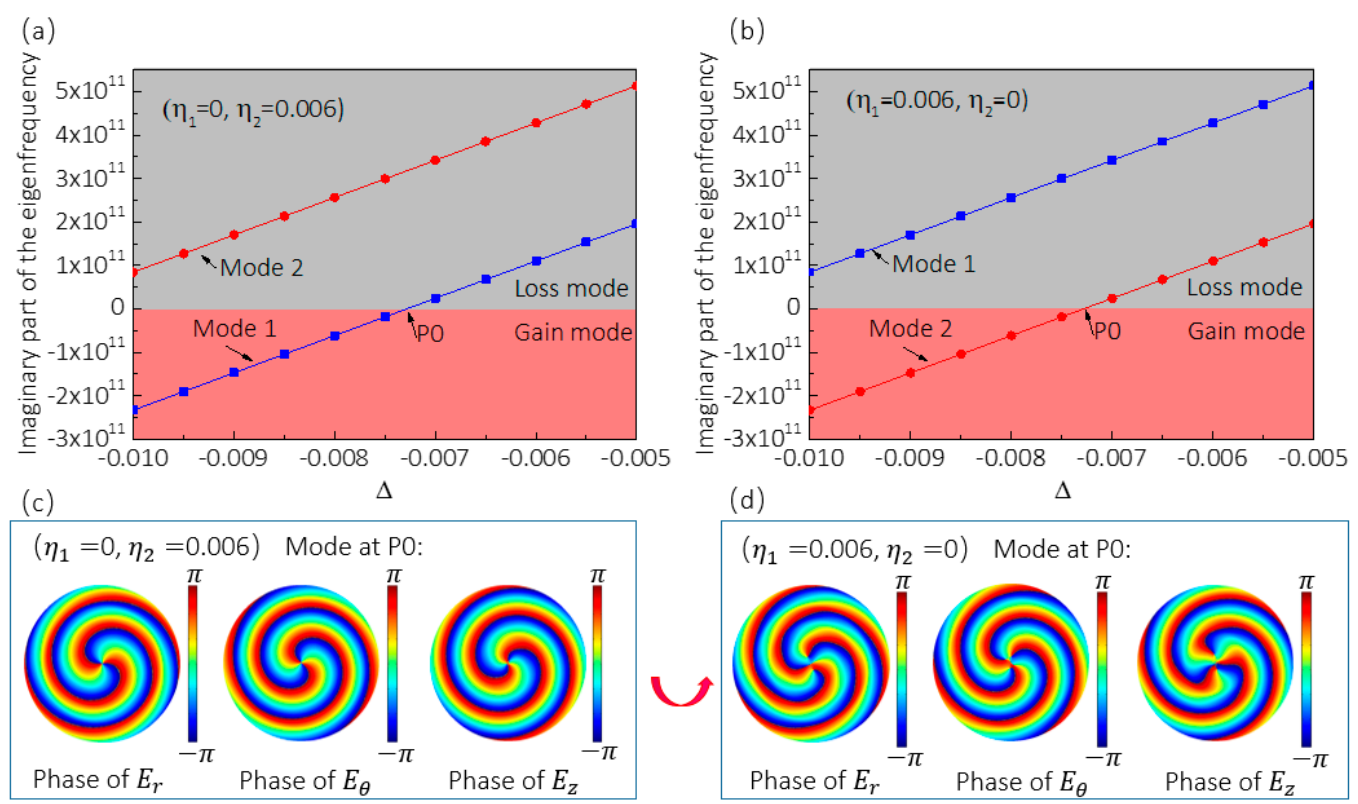

Figure 4. Switching the OAM carried by the emitted light. (a), (b), The homogeneous background gain dependence of the imaginary part of the eigenfrequencies of modes for the system with parameters $\left(\eta_{1}=0, \eta_{2}=0.006\right)(\mathbf{a})$, and $\left(\eta_{1}=0.006, \eta_{2}=0\right)(\mathbf{b})$. (c) The emitted lasing beam corresponding to P0 in (a). (d) The emitted lasing beam corresponding to P0 in (b). The phases of the emission modes in (c) and (d) are in the cross section (the radius of the area is $5000 \mathrm{~nm}$ ) of the emission mode at $2 \mu \mathrm{m}$ above the upper surface of the cavity.

\section{Conclusions}

In this paper, we propose a mechanism for constructing OAM light by modulating the scattered waves from the standing-wave WGM in the plasmonic ring cavity. Within this framework, the single-mode lasing can be realized. Moreover, the OAM of the emitted light can be designed on demand. This work provides a mechanism for exploring a single-mode OAM laser with tunable OAM at the microscale.

Author Contributions: Conceptualization, X.W.; writing—original draft, X.W.; writing-review and editing, X.W., X.H. and T.Z.; supervision, X.W., X.H. and T.Z. All authors have read and agreed to the published version of the manuscript.

Funding: This research was funded by National Natural Science Foundation of China (Grant Nos. 61822501, and 11734001), and the Beijing Natural Science Foundation (Grant No. Z180015).

Data Availability Statement: The data presented in this study are available within the article.

Conflicts of Interest: The authors declare no conflict of interest.

\section{References}

1. Allen, L.; Beijersbergen, M.W.; Spreeuw, R.J.C.; Woerdman, J.P. Orbital angular momentum of light and the transformation of Laguerre-Gaussian laser modes. Phys. Rev. A 1992, 45, 8185-8189. [CrossRef]

2. Bozinovic, N.; Yue, Y.; Ren, Y.; Tur, M.; Kristensen, P.; Huang, H.; Willner, A.E.; Ramachandran, S. Terabit-scale orbital angular momentum mode division multiplexing in fibers. Science 2013, 340, 1545-1548. [CrossRef] [PubMed]

3. Nicolas, A.; Veissier, L.; Giner, L.; Giacobino, E.; Maxein, D.; Laurat, J. A quantum memory for orbital angular momentum photonic qubits. Nat. Photonics 2014, 8, 234-238. [CrossRef]

4. Maiman, T.H. Stimulated Optical Radiation in Ruby Masers. Nature 1960, 187, 493-494. [CrossRef]

5. Khajavikhan, M.; Simic, A.; Katz, M.; Lee, J.H.; Slutsky, B.; Mizrahi, A.; Lomakin, V.; Fainman, Y. Thresholdless nanoscale coaxial lasers. Nature 2012, 482, 204-207. [CrossRef]

6. Lubatsch, A.; Frank, R. A Self-Consistent Quantum Field Theory for Random Lasing. Appl. Sci. 2019, 9, 2477. [CrossRef]

7. Lubatsch, A.; Frank, R. Quantum Many-Body Theory for Exciton-Polaritons in Semiconductor Mie Resonators in the NonEquilibrium. Appl. Sci. 2020, 10, 1836. [CrossRef] 
8. Okamoto, T.; Mori, M. Random Laser Action in Dye-Doped Polymer Media with Inhomogeneously Distributed Particles and Gain. Appl. Sci. 2019, 9, 3499. [CrossRef]

9. Zhang, W.X.; Xie, X.; Hao, H.M.; Dang, J.C.; Xiao, S.; Shi, S.S.; Ni, H.Q.; Niu, Z.C.; Wang, C.; Jin, K.J.; et al. Low-threshold topological nanolasers based on the second-order corner state. Light Sci. Appl. 2020, 9, 109. [CrossRef]

10. Schneider, C.; Rahimi-Iman, A.; Kim, N.Y.; Fischer, J.; Savenko, I.G.; Amthor, M.; Lermer, M.; Wolf, A.; Worschech, L.; Kulakovskii, V.D.; et al. An electrically pumped polariton laser. Nature 2013, 497, 348-352. [CrossRef]

11. Ye, Z.Y.; Su, M.; Li, J.N.; Jing, C.N.; Xu, S.B.; Liu, L.Q.; Ren, G.C.; Wang, X.L. Laser nano-technology of light materials: Precision and opportunity. Opt. Laser Technol. 2021, 139, 106988. [CrossRef]

12. Al-Shibaany, Z.Y.A.; Penchev, P.; Hedley, J.; Dimov, S. Laser Micromachining of Lithium Niobate-Based Resonant Sensors towards Medical Devices Applications. Sensors 2020, 20, 2206. [CrossRef]

13. Shen, Y.; Wang, X.J.; Xie, Z.W.; Min, C.J.; Fu, X.; Liu, Q.; Gong, M.; Yuan, X.C. Optical vortices 30 years on: OAM manipulation from topological charge to multiple singularities. Light Sci. Appl. 2019, 8, 90. [CrossRef]

14. Zeng, J.; Li, L.; Yang, X.; Gao, J. Generating and separating twisted light by gradient-rotation split-ring antenna metasurfaces. Nano Lett. 2016, 16, 3101-3108. [CrossRef]

15. Zeng, J.; Gao, J.; Luk, T.S.; Litchinitser, N.M.; Yang, X. Structuring light by concentric-ring patterned magnetic metamaterial cavities. Nano Lett. 2015, 15, 5363-5368. [CrossRef] [PubMed]

16. Kotlyar, V.V.; Almazov, A.A.; Khonina, S.N.; Soifer, V.A.; Elfstrom, H.; Turunen, J. Generation of phase singularity through diffracting a plane or Gaussian beam by a spiral phase plate. J. Opt. Soc. Am. A 2005, 5, 849-861. [CrossRef] [PubMed]

17. Khonina, S.N.; Podlipnov, V.V.; Karpeev, S.V.; Ustinov, A.V.; Volotovsky, S.G.; Ganchevskaya, S.V. Spectral control of the orbital angular momentum of a laser beam based on 3D properties of spiral phase plates fabricated for an infrared wavelength. Opt. Express 2020, 12, 18407-18417. [CrossRef]

18. Li, H.L.; Phillips, D.B.; Wang, X.Y.; Ho, Y.-L.D.; Chen, L.; Zhou, X.Q.; Zhu, J.B.; Yu, S.Y.; Cai, X.L. Orbital angular momentum vertical-cavity surface-emitting lasers. Optica 2015, 2, 547-552. [CrossRef]

19. Padgett, M.; Courtial, J.; Allen, L. Light's orbital angular momentum. Phys. Today 2004, 57, 35-40. [CrossRef]

20. Marrucci, L.; Manzo, C.; Paparo, D. Optical spin-to-orbital angular momentum conversion in inhomogeneous anisotropic media. Phys. Rev. Lett. 2006, 96, 163905. [CrossRef] [PubMed]

21. Yu, N.; Genevet, P.; Kats, M.; Aieta, F.; Tetienne, J.; Capasso, F.; Gaburro, Z. Light propagation with phase discontinuities: Generalized laws of reflection and refraction. Science 2011, 334, 333-337. [CrossRef]

22. Yang, Y.; Wang, W.; Moitra, P.; Kravchenko, I.I.; Briggs, D.P.; Valentine, J. Dielectric meta-reflectarray for broadband linear polarization conversion and optical vortex generation. Nano Lett. 2014, 14, 1394-1399. [CrossRef] [PubMed]

23. Heckenberg, N.R.; McDuff, R.; Smith, C.P.; White, A.G. Generation of optical phase singularities by computer-generated holograms. Opt. Lett. 1992, 17, 221-223. [CrossRef] [PubMed]

24. Senatsky, Y.; Bisson, J.; Li, J.; Shirakawa, A.; Thirugnanasambandam, M.; Ueda, K. Laguerre-Gaussian modes selection in diode-pumped solid-state lasers. Opt. Rev. 2012, 19, 201-221. [CrossRef]

25. Oron, R.; Davidson, N.; Friesem, A.A.; Hasman, E. Efficient formation of pure helical laser beams. Opt. Commun. 2000, 182, 205-208. [CrossRef]

26. Oron, R.; Danziger, Y.; Davidson, N.; Friesem, A.A.; Hasman, E. Laser mode discrimination with intra-cavity spiral phase elements. Opt. Commun. 1999, 169, 115-121. [CrossRef]

27. Bisson, J.-F.; Senatsky, Y.; Ueda, K. Generation of Laguerre-Gaussian modes in Nd: YAG laser using diffractive optical pumping. Laser Phys. Lett. 2005, 2, 327-333. [CrossRef]

28. Okida, M.; Omatsu, T.; Itoh, M.; Yatagai, T. Direct generation of high power Laguerre-Gaussian output from a diode-pumped Nd: YVO 4 1.3- $\mu \mathrm{m}$ bounce laser. Opt. Express 2007, 15, 7616-7622. [CrossRef]

29. Caley, A.J.; Thomson, M.J.; Liu, J.; Waddie, A.J.; Taghizadeh, M.R. Diffractive optical elements for high gain lasers with arbitrary output beam profiles. Opt. Express 2007, 15, 10699-10704. [CrossRef]

30. Ito, A.; Kozawa, Y.; Sato, S. Generation of hollow scalar and vector beams using a spot-defect mirror. J. Opt. Soc. Am. A 2010, 27, 2072-2077. [CrossRef] [PubMed]

31. Kano, K.; Kozawa, Y.; Sato, S. Generation of purely single transverse mode vortex beam from a He-Ne laser cavity with a spot-defect mirror. Int. J. Opt. 2011, 2012, 359141. [CrossRef]

32. Naidoo, D.; Roux, F.S.; Dudley, A.; Litvin, I.; Piccirillo, B.; Marrucci, L.; Forbes, A. Controlled generation of higher-order Poincaré sphere beams from a laser. Nat. Photon. 2016, 10, 327-332. [CrossRef]

33. Al-Attili, A.Z.; Burt, D.; Li, Z.; Higashitarumizu, N.; Gardes, F.Y.; Oda, K.; Ishikawa, Y.; Saito, S. Germanium vertically lightemitting micro-gears generating orbital angular momentum. Opt. Express 2018, 26, 34675-34688. [CrossRef] [PubMed]

34. Miao, P.; Zhang, Z.; Sun, J.; Walasik, W.; Longhi, S.; Litchinitser, N.M.; Feng, L. Orbital angular momentum microlaser. Science 2016, 353, 464-467. [CrossRef] [PubMed]

35. Feng, L.; Ayache, M.; Huang, J.; Xu, Y.-L.; Lu, M.-H.; Chen, Y.-F.; Fainman, Y.; Scherer, A. Nonreciprocal light propagation in a silicon photonic circuit. Science 2011, 333, 729-733. [CrossRef] 